\title{
Spectral patterns and ultrafast dynamics in planar acetylene
}

\author{
M.E. Kellman ${ }^{\mathrm{a}}$, J.P. Rose, and V. Tyng \\ Department of Chemistry, University of Oregon, Eugene, OR 97403, USA
}

Received 29 August 2000 and Received in final form 14 September 2000

\begin{abstract}
A diabatic correlation diagram procedure is used to classify energy and intensity patterns in a planar model for coupled stretches and bends of acetylene using an effective spectroscopic fitting Hamiltonian. Analysis with polyad phase spheres accounts for the observed patterns in terms of classical phase space structure and bifurcations of the normal modes.
\end{abstract}

PACS. 31.15.-p Calculations and mathematical techniques in atomic and molecular physics (excluding electron correlation calculations)

\section{Introduction}

This paper describes using analysis of frequency domain spectra to obtain information about ultrafast dynamics in acetylene. In a recent paper [1], we demonstrated that there are novel energy and intensity patterns in highly excited bending spectra of acetylene when the usual normal modes quantum number classification breaks down. These patterns are identified using a diabatic correlation diagram technique developed and applied previously [2] to $\mathrm{H}_{2} \mathrm{O}$, with a very partial preliminary account given for application to acetylene [3]. An approximate assignment with effective quantum numbers results, giving a classification into subpolyads, or sequences. These can be fit with an effective Hamiltonian and analyzed in a classical phase space picture, giving an account of observed energy and intensity patterns that goes well beyond the earlier identification [4-11] of polyads.

In this paper we take an approach similar to reference [1] for spectral patterns of a model Hamiltonian for $\mathrm{C}_{2} \mathrm{H}_{2}$ which includes the stretches as well as bends, but in a model confined to the plane. There are several reasons for starting with a simplified planar model. When the stretches are introduced, the stretch-bend problem in the plane is essentially as complicated as the threedimensional pure bends problem: both involve three nonlinear resonance coupling terms. Furthermore, the planar model has been of interest in itself, in model studies of coherent control [12]. Lastly, a better understanding of the stretch-bend problem, even in a simplified planar model, is of pressing interest, because of the possible involvement of the stretches in the acetylene-vinylidene isomerization process. The isomerization has been a focus of interest of the pure-bends spectral analysis [13], yet there is reason to think from $a b$ initio calculations [14] that the transition state in the isomerization may involve the stretches as well as the bends.

\footnotetext{
a e-mail: Kellman@Oregon.Uoregon. Edu
}

Here we present an exploratory study to see if the kinds of spectral analysis given in [1] for the pure bends spectrum will hold for the stretch-bend system as well - an expectation that we will see is fully met. Successful results here for the planar stretch-bend system and in reference [1] for the three-dimensional bends motivate for the future a full-scale three-dimensional approach for the entire spectral system of $\mathrm{C}_{2} \mathrm{H}_{2}$ stretches and bends.

We will make use of earlier work $[15,16]$ on single resonance systems for interpretation of the multiresonance planar dynamics. In reference [15], we performed a bifurcation analysis of highly excited acetylene bends coupled by a single Darling-Dennison resonance. This was a simplified, single resonance model which showed that the bend spectra exhibit a new type of correlated motion called precessional modes [17], in addition to the more familiar normal and local modes. In reference [16], we performed a similar analysis of the "2345" resonance between bends, $\mathrm{C}-\mathrm{C}$ stretch, and antisymmetric $\mathrm{C}-\mathrm{H}$ stretch. The 2345 resonance comes into play in the present work, but only as a "bystander" to the bifurcation analysis of the DarlingDennison resonance between the bends.

\section{Spectroscopic Hamiltonian}

The spectroscopic Hamiltonian for the planar stretchbend model for vibrations of acetylene consists of a diagonal part, $\hat{\mathbf{H}}_{0}$, and an off-diagonal part, $\hat{\mathbf{V}}$ :

$$
\hat{\mathbf{H}}=\hat{\mathbf{H}}_{0}+\hat{\mathbf{V}} .
$$

The diagonal part contains the harmonic and anharmonic contributions to the vibrational energy levels,

$$
\hat{\mathbf{H}}_{0}=\sum_{j=1}^{5} \omega_{j} n_{j}+\sum_{j=1}^{5} \sum_{k=j}^{5} \chi_{j k} n_{j} n_{k}
$$




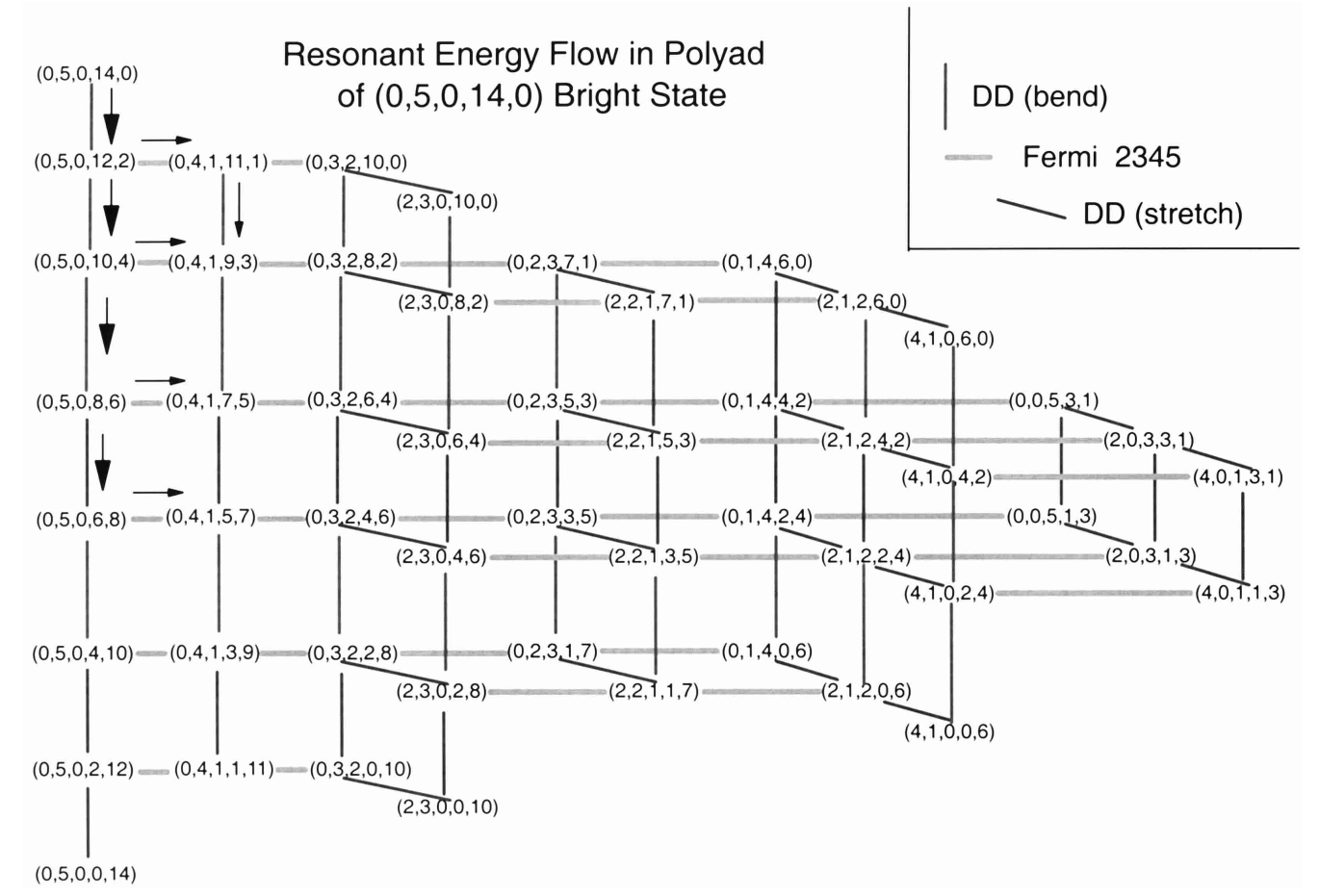

Fig. 1. Resonance lattice of the Darling-Dennison bends coupling $\hat{\mathbf{V}}_{\text {DD-bend }}$, Darling-Dennison stretch coupling $\hat{\mathbf{V}}_{\mathrm{DD}-\text { stretch }}$, and 2345 Fermi resonance coupling $\hat{\mathbf{V}}_{2345}$, used in planar acetylene model of equation (3).

Table 1. Parameters (in $\mathrm{cm}^{-1}$ ) for diagonal terms and resonance couplings of planar Hamiltonian; see Section 2.

\begin{tabular}{cccc}
\hline$\omega_{1}=3398.74 \mathrm{~cm}^{-1}$ & $\chi_{12}=-12.62$ & $\chi_{24}=-12.48$ & $\chi_{45}=-2.406$ \\
$\omega_{2}=1981.71$ & $\chi_{13}=-105.09$ & $\chi_{25}=-1.57$ & $\chi_{55}=-2.335$ \\
$\omega_{3}=3316.09$ & $\chi_{14}=-15.58$ & $\chi_{33}=-27.41$ & $g_{44}=0.759$ \\
$\omega_{4}=609.016$ & $\chi_{15}=-10.85$ & $\chi_{34}=-6.96$ & $g_{45}=6.541$ \\
$\omega_{5}=729.170$ & $\chi_{22}=-7.39$ & $\chi_{35}=-8.69$ & $g_{55}=3.49$ \\
$\chi_{11}=-25.87$ & $\chi_{23}=-6.10$ & $\chi_{44}=3.082$ & \\
\hline$K_{\text {DD-stretch }}=105.83$ & $K_{2345}=18.28$ & $K_{\text {DD-bend }}=10.00$ & \\
\hline
\end{tabular}

where $\omega_{j}$ is the harmonic frequency for mode $j ; n_{j}$ is the number of vibrational quanta in mode $j$; and $\chi_{j k}$ is the anharmonicity constant between modes $j$ and $k$.

The off-diagonal part $\hat{\mathbf{V}}$ contains the spectroscopically significant resonance couplings, i.e. those needed to fit the data to the desired level of detail and accuracy. Parameters for the coupling strengths are determined in the fitting of the spectrum.

Many resonances have been detected in acetylene spectra using various excitation techniques [18-21]. With bends-only excitation, all the spectroscopically important couplings turn out to be among states with quanta only in the bend modes $[15,22]$. For pure bends spectra, important couplings are of the Darling-Dennison type [23]. In the planar model, there is only one of these, which we call $\hat{\mathbf{V}}_{\text {DD-bend. With excitation of the } \mathrm{C}-\mathrm{C} \text { stretch as }}$ well as the bends, coupling is possible to the $\mathrm{C}-\mathrm{H}$ stretch modes. The most important of these is the 2345 resonance coupling $[18,19] \hat{\mathbf{V}}_{2345}$, which transfers one quantum each of the $\mathrm{C}-\mathrm{C}$ stretch $\nu_{2}$, trans bend $\nu_{4}$, and cis bend $\nu_{5}$ to the antisymmetric $\mathrm{C}-\mathrm{H}$ stretch $\nu_{3}$. Then with involvement of the $\mathrm{C}-\mathrm{H}$ stretch $\nu_{3}$ via $\hat{\mathbf{V}}_{2345}$, a third important coupling is the Darling-Dennison resonance between the stretches, $\hat{\mathbf{V}}_{\text {DD-stretch, typically extremely prominent in }}$ coupled stretch systems, because of its connection to the transition from normal to local modes $[31,32]$. The stretchbend Hamiltonian then is

$$
\begin{aligned}
\hat{\mathbf{H}}_{s b}=\hat{\mathbf{H}}_{0} & +\hat{\mathbf{V}}_{\mathrm{DD}-\text { stretch }}+\hat{\mathbf{V}}_{\mathrm{DD}-\text { bend }}+\hat{\mathbf{V}}_{2345} \\
\hat{\mathbf{V}}_{\mathrm{DD}-\text { bend }} & =K_{\mathrm{DD}-\text { bend }}\left[\mathbf{a}_{4}^{\dagger} \mathbf{a}_{4}^{\dagger} \mathbf{a}_{5} \mathbf{a}_{5}+\text { h.c. }\right] \\
\hat{\mathbf{V}}_{\mathrm{DD}-\text { stretch }} & =K_{\mathrm{DD}-\text { stretch }}\left[\mathbf{a}_{1}^{\dagger} \mathbf{a}_{1}^{\dagger} \mathbf{a}_{3} \mathbf{a}_{3}+\text { h.c. }\right] \\
\hat{\mathbf{V}}_{2345} & =K_{2345}\left[\mathbf{a}_{2}^{\dagger} \mathbf{a}_{3} \mathbf{a}_{4}^{\dagger} \mathbf{a}_{5}^{\dagger}+\text { h.c. }\right] .
\end{aligned}
$$

The action of the three coupling operators in (3) is depicted schematically in the "resonance lattice" of Figure 1. The values for the spectroscopic parameters, including the strengths of the three resonance couplings as well as the diagonal terms of equation (2), are given in Table 1 . These parameters are those reported by Jonas et al. [24], and 


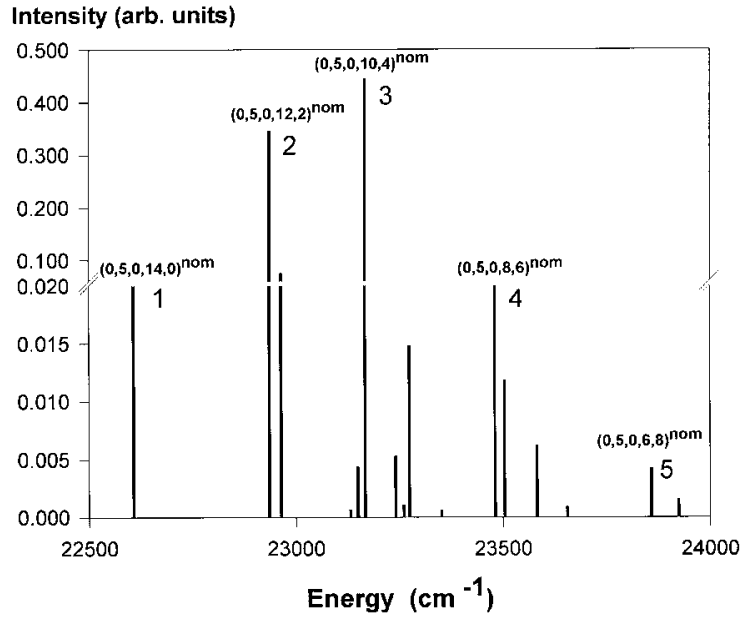

Fig. 2. Simulated spectrum of acetylene polyad originating from zero-order bright state $(0,5,0,14,0)=\left(n_{1}, n_{2}, n_{3}, n_{4}, n_{5}\right)$ in five-mode planar model of Hamiltonian (3). Five of the levels assigned to the "primary" subpolyad by the diabatic correlation diagram method are numbered 1-5 with their assignment in terms of nominal quantum numbers indicated.

subsequently modified very slightly by Jonas [25]. (The parameters differ from those used in reference [1] solely because of the suppression of zero-point terms in the quantum numbers.)

\section{Patterns in the coupled stretch-bend spectrum}

Figure 2 shows a simulation of a part of a dispersed fluorescence (DF) spectrum using the Hamiltonian (3). In DF experiments, a transition to an upper electronic state is followed by a Franck-Condon transition back to the ground state, resulting in a wave packet-like vibrational excitation on the ground state, i.e. a superposition of "bright" excited zero-order vibrational states. Experimental DF spectra of $\mathrm{C}_{2} \mathrm{H}_{2}$ with all the excitation in $\nu_{2}$ and the transbend $\nu_{4}$ have been observed by Field and coworkers $[6-8$, 10,24]. They have analyzed these spectra into polyads of levels, each polyad labeled by $n_{2}$ and $n_{4}$, the quantum numbers of the zero-order bright state from which the polyad originates. Algebraic analysis [4-11] shows that the polyad numbers are good quantum numbers for the spectroscopic Hamiltonian, which therefore is block-diagonal. Figure 2 shows a stick spectrum of the polyad originating from the zero-order bright state $(0,5,0,14,0)$ calculated in the planar model of (3); this is the spectrum for which we will attempt to detect and interpret spectral patterns in this paper. The intensities are calculated from the eigenvectors of the Hamiltonian. The intensities within the polyad are assumed to originate from the single zeroorder bright state $(0,5,0,14,0)$ which labels the polyad. The intensity of each level is determined by the contribution of the polyad bright state to the given eigenvector. It is an assumption that each polyad originates from a single zero-order bright state; however this simplification has proven empirically [6-8] to be valid for $\mathrm{C}_{2} \mathrm{H}_{2}$ dispersed fluorescence spectra, similar to the spectra in the planar model considered here.

The initial intensity in the bright state "fractionates" into intensities like those in Figure 2 because of the resonance couplings, which break the $\nu_{2}$ and $\nu_{4}$ quantum numbers. Inspection of Figure 2 suggests patterns within the polyad. It appears that there might be a sequence of especially intense levels, as well as one or more sequences of less intense levels.

If so, these patterns must be understood in terms of the resonance couplings in the Hamiltonian $\hat{\mathbf{H}}_{s b}$ of (3). The action of the three coupling operators is depicted schematically in the "resonance lattice" of Figure 1. From Table 1, the Darling-Dennison stretch resonance $\hat{\mathbf{V}}_{\mathrm{DD} \text {-stretch has }}$ by far the largest coupling parameter. However, as seen in the resonance lattice, the Darling-Dennison bends coupling $\hat{\mathbf{V}}_{\text {DD-bend }}$ is "primary", in the sense that it provides the direct energy transfer mechanism from the zero-order bright state $\left(0, n_{2}, 0, n_{4}, 0\right)$. As such, it is expected to dominate the intensity pattern of the spectrum, a fact which will play a key role in the analysis to follow. The stretchstretch coupling $\hat{\mathbf{V}}_{\text {DD-stretch }}$ actually plays a minor role in the intensities, because it is mediated by the coupling $\hat{\mathbf{V}}_{2345}$.

To unravel these competing effects, we use the diabatic correlation diagram technique, which has proven [1-3,27] to be a useful systematic tool to identify and classify patterns such as those in Figure 2.

\section{Diabatic correlation assignment}

The diabatic correlation technique $[1,2]$ is an empirical procedure for assignment of a complete, though approximate and non-rigorous, set of effective or "nominal" quantum numbers. A set of zero-order levels with a complete set of $N$ good quantum numbers $\left(n_{1}, \ldots, n_{N}\right)$ is followed along diabatic curves as a quantum number-breaking perturbation is gradually turned on - here, the resonance terms of (3). The zero-order quantum numbers are carried along as labels for the diabatic levels, so at each point we possess a set of $N$ labels for each state. To the extent that these labels have physical meaning, we have an assignment of each level in terms of a set of effective or nominal quantum numbers $\left(n_{1}, \ldots, n_{N}\right)^{\text {nom }}$. These quantum numbers are used to group levels of a polyad into sets of subpolyads, or sequences, that we expect will bring out patterns in the energies and intensities.

Figure 2 shows the stick spectrum for polyad $(0,5,0,14,0)$. The energy levels of this spectrum are calculated from the Hamiltonian (3), with the assumptions discussed in the preceding section. We focus on the subpolyad of 8 levels with diabatic assignments $(0,5,0,14,0)^{\text {nom }},(0,5,0,12,2)^{\text {nom }}, \ldots,(0,5,0,0,14)^{\text {nom }}$.

We call this the "primary" subpolyad because it originates from the primary coupling pathway out of the zeroorder bright state induced by the Darling-Dennison bend resonance $\hat{\mathbf{V}}_{\text {DD-bend }}$, as depicted in the preceding section 

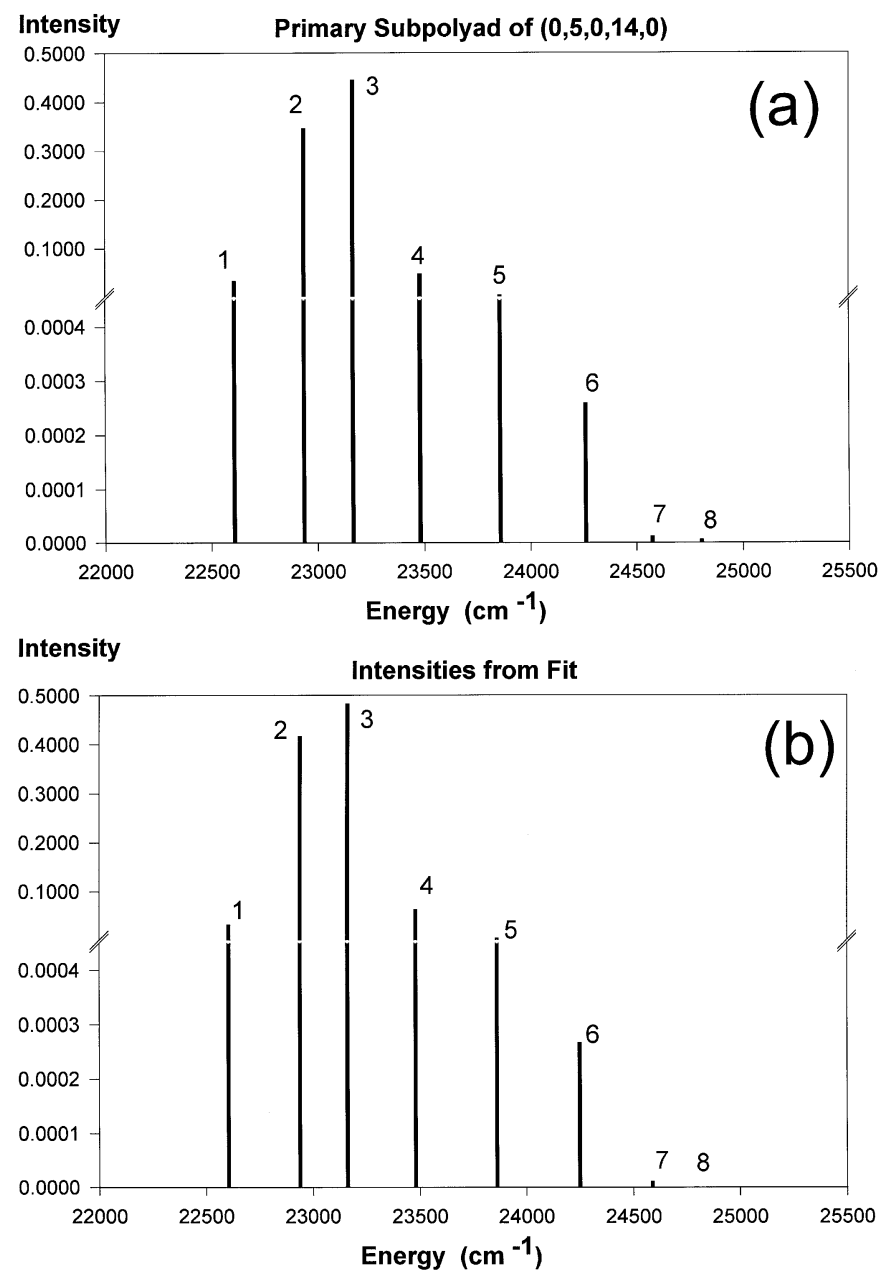

Fig. 3. (a) The eight levels of the primary subpolyad of acetylene polyad of Figure 2, see Section 4; (b) energies and intensities of the fit for the primary subpolyad, see Section 5 .

in connection with the resonance lattice of Figure 1. The levels from the primary subpolyad with sufficient intensity to be visible in Figure 2 are numbered, and labeled with their effective or nominal quantum number assignments $\left(n_{1}, \ldots, n_{5}\right)^{\text {nom }}$.

The calculated spectrum of the entire subpolyad is shown by itself on an expanded scale in Figure 3a. Two patterns are evident. First, the energy spacing of adjacent levels has a minimum between levels 2 and 3 . This is reminiscent of the minimum in spacing between levels $[28,29]$ that corresponds semiclassically to tori that lie on opposite sides of a phase space separatrix. Second, the intensity is at a maximum for levels 2 and 3. Do these patterns really have an origin in the molecular dynamics as understood in terms of classical phase space structure?

\section{Fit of the primary subpolyad}

To answer, this, we have fit the primary subpolyad with a very simple single resonance Hamiltonian:

$$
\begin{aligned}
\hat{\mathbf{H}}_{\mathrm{fit}}=\omega_{4}^{0} n_{4}+\omega_{5}^{0} n_{5}+\chi_{44} n_{4}^{2}+ & \chi_{45} n_{4} n_{5}+\chi_{55} n_{5}^{2} \\
& +K\left[a_{4}^{\dagger} a_{4}^{\dagger} a_{5} a_{5}+\text { h.c. }\right] .
\end{aligned}
$$

In a fit of subpolyads from various polyads, i.e. with variable polyad number, the form (5) has five independent diagonal parameters plus the coupling parameter $K$. An alternative is to fit the primary subpolyad of just a single polyad, in which case (5) has only three independent diagonal parameters plus the coupling parameter $K$. We reported a fit for multiple polyads in reference [1], where we analyzed together the primary subpolyads of all the polyads that were fit. Here we present analysis of just one polyad, based on the fit of its primary subpolyad alone. Hence, we have a four-parameter fit of the 8 levels of the primary subpolyad of $(0,5,0,14,0)$. In itself, this is hardly a very stringent fit. However, this leaves seven independent intensities (with one arbitrary overall intensity factor) as true predictions of the fit. The test of the cogency of the spectral assignment and fitting procedure is then the prediction of intensities, along with the interpretation in the next section of the energy and intensity patterns in terms of phase space structure. The results of the fit for the both the energies and intensities, shown in Figure 3b, are obviously very good.

\section{Polyad phase sphere}

We used the fit to construct a polyad phase sphere [32-34] for the primary subpolyad, shown in Figure 4 . The phase sphere is a way of representing and visualizing the spectrum in phase space. The phase sphere was first developed for a system of two modes coupled by a single resonance [32]; didactic presentations can be found in references [33, 34]. The phase sphere projects out the conserved polyad number and its conjugate angle, leaving a semiclassical representation of each energy level of a polyad on a reduced phase space, which is properly represented on a surface of spherical topology $[35,36]$.

The sphere in Figure 4 shows considerable phase space structure, with separatrices dividing regions of vibrational motion of different character. The three kinds of motion observed are local and normal bend modes, and the "precessional modes" identified [15] in a single resonance model of the acetylene bends as a new kind of vibrational motion for the Darling-Dennison Hamiltonian. The sphere has a separatrix at the north pole, dividing normal and precessional modes regions. Near the south pole, another separatrix divides the normal region from a tiny local modes region.

How well does the phase sphere account for the observed energy and intensity patterns in Figure 3?

A classical separatrix is predicted [28] to induce a minimum in the level spacing. The phase sphere of Figure 4 


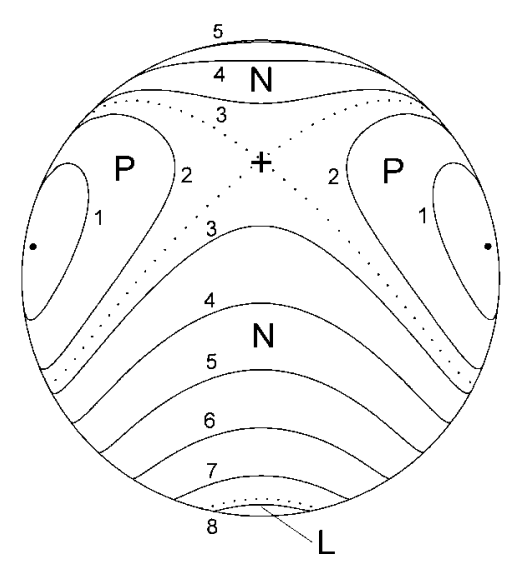

Fig. 4. Polyad phase sphere from fit of primary subpolyad of polyad from fractionation of zero-order bright state $(0,5$, $0,14,0)$ of acetylene. Fit is to single resonance Hamiltonian, described in Section 5. Each quantum level is represented semiclassically on the sphere as a trajectory. Notation: N normal; $\mathrm{L}$ local; $\mathrm{P}$ precessional. The + is the north pole, which semiclassically corresponds to the pure zero-order bright state $(0$, $5,0,14,0)$ without zero-point motion. The points near the east and west extremities are stable fixed points, corresponding to the precessional mode overtones.

therefore predicts a minimum in spacing between levels 2 , 3 because they are on opposite sides of separatrix. This is just what is seen in Figure 3.

Next, we consider the intensities. In the zero-order phase sphere, a circumpolar trajectory near the north pole corresponds to the quantum zero-order bright state. In the phase sphere in Figure 4, the states corresponding to trajectories nearest the north pole are expected to have the greatest intensity. This is just what is seen: levels 2 , 3 are nearest the north pole, and they have the greatest intensities. Furthermore, the intensity is predicted to be fractionated strongly among all the states of the subpolyad, because the north pole, corresponding to the zeroorder bright state in $\hat{\mathbf{H}}_{0}$, lies right at an unstable fixed point defining the separatrix in Figure 4; the phase sphere is strongly divided because of the profound effect of the Darling-Dennison bend coupling in $\hat{\mathbf{H}}_{\text {fit }}$. This fractionation with intensities descending from levels 2,3 is exactly what is observed in Figure 4.

\section{Conclusions and outlook}

We have shown that spectral patterns directly related to elementary modes of vibration at high energy can be identified in the planar stretch-bend multiresonance model of acetylene with the use of effective quantum number assignments from diabatic correlation diagrams. We can reproduce these patterns with an effective DarlingDennison resonance Hamiltonian. This leads to a polyad phase sphere which accounts very well for the detailed energy and intensity patterns in terms of ideas of phase space structure and bifurcations of normal modes.
There is no reason to think that these energy and intensity patterns and a coherent account of them should be limited to the primary subpolyads. An attempt to treat the complete spectrum - not just the primary subpolyads - along the lines developed here appears highly desirable.

However, this will require methods that go beyond those used here. The fit of (5) used a zero-order energy formula given in the standard way by terms linear and quadratic in the zero-order quantum numbers. The price we pay for using the simplified zero-order energy formula (5) is that we can only fit a subset of levels of the entire spectrum - otherwise a multiresonance fitting Hamiltonian like $\hat{\mathbf{H}}_{s b}$ of (3) would not have been necessary in the first place.

Simultaneously with the work of this paper and of reference [1], we have been developing a "dressed basis" approach [27] for complex spectra of highly excited molecular vibrations. In the dressed basis method, a zero-order basis is dressed by all the resonance couplings in the effective Hamiltonian, except for a residual coupling $\hat{\mathbf{V}}_{\mathrm{i}}$. Acting within subpolyads of the dressed basis, an effective coupling $\hat{\mathbf{V}}_{\mathrm{i}}^{\text {eff }}$ is defined in correspondence to the residual $\hat{\mathbf{V}}_{\mathrm{i}}$. The goal of the dressed basis is to incorporate as much as possible the effects of nonintegrability and chaos, while retaining much of the simplicity of integrable systems via the effective residual single resonance coupling $\hat{\mathbf{V}}_{\mathrm{i}}^{\text {eff }}$. Numerical testing [27] shows that the procedure works remarkably well.

We regard the fit here of the primary subpolyad to the simplified form (5) as incorporating some aspects of a full dressed basis analysis. If successful, a dressed basis fit of the entire spectrum should be useful in conjunction with the diabatic correlation technique in illuminating the complete spectral pattern, as well as connections with concurrent bifurcation analysis of the spectroscopic Hamiltonian. Steps toward bifurcation analysis for various subsystems of acetylene have been taken by several authors [41-43]. The bifurcation analysis of the full threedimension stretch-bend acetylene system is clearly feasible along the analytical lines developed previously [34,38-40]. A further challenge will be to use the acetylene dressed basis and bifurcation analysis to elucidate the pathway of the acetylene-vinylidene isomerization. The power of bifurcation analysis of spectra for ultrafast intramolecular rearrangement processes has already been demonstrated for the isomerization spectrum of HCP [29].

This work was supported by the Department of Energy Basic Energy Sciences program under Contract DE-FG0398ER14848.

\section{References}

1. J.P. Rose, M.E. Kellman, J. Phys. Chem. A (in press).

2. J.P. Rose, M.E. Kellman, J. Chem. Phys. 105, 7348 (1996).

3. M.E. Kellman, Adv. Chem. Phys. 101, 590 (1997).

4. M.E. Kellman, J. Chem. Phys. 93, 6330 (1990).

5. M.E. Kellman, G. Chen, J. Chem. Phys. 95, 8671 (1991). 
6. S.A.B. Solina, J.P. O'Brien, R.W. Field, W.F. Polik, Ber. Bunsenges. Phys. Chem. 99, 555 (1995).

7. S.A.B. Solina, J.P. O'Brien, R.W. Field, W.F. Polik, J. Phys. Chem. 100, 7797 (1996).

8. J.P. O'Brien, M.P. Jacobson, J.J. Sokol, S.L. Coy, R.W. Field, J. Chem. Phys. 108, 7100 (1998).

9. M. Abbouti Temsamani, M. Herman, J. Chem. Phys. 102, 6371 (1995).

10. M. Abbouti Temsamani, M. Herman, S.A.B. Solina, J.P. O'Brien, R.W. Field, J. Chem. Phys. 105, 11357 (1996).

11. M.I. El Idrissi, J. Lievin, A. Campargue, M. Herman, J. Chem. Phys. 110, 2074 (1999).

12. L. Liu, J.T. Muckerman, J. Chem. Phys. 110, 2446 (1999).

13. M.P. Jacobson, R.W. Field, J. Phys. Chem. A 104, 3073 (2000).

14. N. Chang, M. Shen, C. Yu, J. Chem. Phys. 106, 3237 (1997).

15. J.P. Rose, M.E. Kellman, J. Chem. Phys. 105, 10743 (1996).

16. J.P. Rose, M.E. Kellman, J. Chem. Phys. 103, 7255 (1995).

17. S.K. Gray, M.S. Child, Mol. Phys. 53, 961 (1984).

18. W.J. Lafferty, R.J. Thibault, J. Mol. Spectrosc. 14, 79 (1964).

19. J. Pliva, J. Mol. Spec. 44, 165 (1972).

20. B.C. Smith, J.S. Winn, J. Chem. Phys. 89, 4638 (1988).

21. B.C. Smith, J.S. Winn, J. Chem. Phys. 94, 4120 (1991).

22. M.P. Jacobson, J.P. O'Brien, R.J. Silbey, R.W. Field, J. Chem. Phys. 109, 121 (1998).

23. B.T. Darling, D.M. Dennison, Phys. Rev. 57, 128 (1940).

24. D.M. Jonas, S.A.B. Solina, B Rajaram, R.J. Silbey, R.W. Field, K. Yamanouchi, S. Tsuchiya, J. Chem. Phys. 99, 7350 (1993).
25. D.M. Jonas, Ph.D. dissertation, Massachusetts Institute of Technology, 1992.

26. L.E. Fried, G.S. Ezra, J. Chem. Phys. 86, 6270 (1987).

27. M.E. Kellman, M.W. Dow, J. Chem. Phys. (submitted).

28. J. Svitak, Z. Li, J. Rose, M.E. Kellman, J. Chem. Phys. 102, 4340 (1995).

29. M. Joyeux, D. Sugny, V. Tyng, M.E. Kellman, H. Ishikawa, R.W. Field, J. Chem. Phys. 112, 4162 (1999).

30. K.K. Lehmann, J. Chem. Phys. 79, 1098 (1983).

31. M.E. Kellman, J. Chem. Phys. 83, 3843 (1985).

32. L. Xiao, M.E. Kellman, J. Chem. Phys. 90, 6086 (1989).

33. M.E. Kellman, Ann. Rev. Phys. Chem. 46, 395 (1995).

34. M.E. Kellman, Dynamical Analysis of Highly Excited Vibrational Spectra: Progress and Prospects in Molecular Dynamics and Spectroscopy by Stimulated Emission Pumping, edited by H.-L. Dai, R.W. Field (World Scientific, Singapore, 1995).

35. C. Jaffe, J. Chem. Phys. 89, 3395 (1988).

36. M.E. Kellman, E.D. Lynch, J. Chem. Phys. 89, 3396 (1988).

37. H. Ishikawa, C. Nagao, N. Mikami, R.W. Field, J. Chem. Phys. 109, 492 (1998).

38. Z.-M. Lu, M.E. Kellman, Chem. Phys. Lett. 247, 195 (1995).

39. Z.-M. Lu, M.E. Kellman, J. Chem. Phys. 107, 1 (1997).

40. S. Keshavamurthy, G.S. Ezra, J. Chem. Phys. 107, 156 (1997).

41. E.L. Sibert, A.B. McCoy, J. Chem. Phys. 105, 469 (1996).

42. R. Prosmiti, S.C. Farantos, J. Chem. Phys. 103, 3299 (1995).

43. M.P. Jacobson, C. Jung, H.S. Taylor, R.W. Field, J. Chem. Phys. 111, 66 (1999). 